\title{
ARTICLE
}

Clinical Study

\section{The impact of immediate breast reconstruction on the time to delivery of adjuvant therapy: the iBRA-2 study}

Rachel L O'Connell ${ }^{1}$, Tim Rattay ${ }^{2}$, Rajiv V Dave ${ }^{3}$, Adam Trickey ${ }^{4}$, Joanna Skillman ${ }^{5}$, Nicola L. P. Barnes ${ }^{3}$, Matthew Gardiner ${ }^{6,7}$, Adrian Harnett ${ }^{8}$, Shelley Potter $\mathbb{D D}^{4,9}$ and Chris Holcombe ${ }^{10}$ on behalf of the iBRA-2 Steering Group and

the Breast Reconstruction Research Collaborative

BACKGROUND: Immediate breast reconstruction (IBR) is routinely offered to improve quality-of-life for women requiring mastectomy, but there are concerns that more complex surgery may delay adjuvant oncological treatments and compromise longterm outcomes. High-quality evidence is lacking. The iBRA-2 study aimed to investigate the impact of IBR on time to adjuvant therapy.

METHODS: Consecutive women undergoing mastectomy \pm IBR for breast cancer July-December, 2016 were included. Patient demographics, operative, oncological and complication data were collected. Time from last definitive cancer surgery to first adjuvant treatment for patients undergoing mastectomy \pm IBR were compared and risk factors associated with delays explored. RESULTS: A total of 2540 patients were recruited from 76 centres; 1008 (39.7\%) underwent IBR (implant-only [ $n=675,26.6 \%]$; pedicled flaps [ $n=105,4.1 \%]$ and free-flaps $[n=228,8.9 \%])$. Complications requiring re-admission or re-operation were significantly more common in patients undergoing IBR than those receiving mastectomy. Adjuvant chemotherapy or radiotherapy was required by 1235 (48.6\%) patients. No clinically significant differences were seen in time to adjuvant therapy between patient groups but major complications irrespective of surgery received were significantly associated with treatment delays.

CONCLUSIONS: IBR does not result in clinically significant delays to adjuvant therapy, but post-operative complications are associated with treatment delays. Strategies to minimise complications, including careful patient selection, are required to improve outcomes for patients.

British Journal of Cancer (2019) 120:883-895; https://doi.org/10.1038/s41416-019-0438-1

\section{BACKGROUND}

Breast cancer is the most common female cancer worldwide with 1.7 million new cases diagnosed each year. ${ }^{1}$ Despite improvements in treatment, however, mastectomy remains the primary surgical treatment for almost $40 \%$ of women $^{2,3}$ and immediate breast reconstruction (IBR) is offered with the aim of improving quality-of-life. $^{4}$

Although psychosocial outcomes are an important consideration when planning treatment, oncological safety remains paramount. Breast reconstruction is associated with more complications than simple mastectomy, ${ }^{5}$ and concerns have been raised that the increased complication rate may lead to the delay or omission of adjuvant chemotherapy or radiotherapy, ${ }^{6}$ which may compromise oncological outcomes. The clinical significance of short delays is unclear, but two recent large population-based studies have shown that patients experiencing delays of more than 90 days in the delivery of chemotherapy experienced worse overall and cancerspecific survival. ${ }^{7,8}$ Furthermore, a recent meta-analysis suggests a $15 \%$ decrease in overall survival for every four-week delay in the delivery of adjuvant chemotherapy. ${ }^{9}$ Delays to radiotherapy have similarly adverse effects but the time-frames are less wellestablished. A meta-analysis including 21 retrospective breast cancer studies suggested an increased risk of loco-regional recurrence if radiotherapy was delayed by more than 8 weeks following surgery, ${ }^{10}$ but other large cohort studies have demonstrated no deleterious effects with delays of up to 20 weeks. ${ }^{11}$

Evidence regarding the impact of IBR on the delivery of adjuvant therapy, however, is inconsistent. ${ }^{6}$ A recent systematic review ${ }^{6}$ failed to demonstrate a clinically significant delay in the initiation of chemotherapy but included 14 mainly single-centre studies with

\footnotetext{
${ }^{1}$ Department of Breast Surgery, The Royal Marsden NHS Foundation Trust, Downs Road, Sutton, Surrey SM2 5PT, UK; ${ }^{2}$ Department of Cancer Studies, Clinical Sciences Building, University of Leicester, Leicester LE2 2LX, UK; ${ }^{3}$ Nightingale Breast Unit, Manchester University NHS Foundation Trust, Southmoor Road, Manchester M23 9LT, UK; ${ }^{4}$ Population Health Sciences, Bristol Medical School, 39 Whatley Road, Clifton, Bristol BS8 2PS, UK; ${ }^{5}$ Department of Plastic Surgery, University Hospitals Coventry and Warwickshire NHS Trust, Clifford Bridge Road, Coventry CV2 2DX, UK; ${ }^{6}$ Nuffield Department of Orthopaedics, Rheumatology and Musculoskeletal Sciences, University of Oxford, Nuffield Orthopaedic Centre, Windmill Road, Headington, Oxford OX3 7HE, UK; ${ }^{7}$ Department of Surgery and Cancer, Imperial College London, London, UK; ${ }^{8}$ Norfolk and Norwich Hospital, Norwich, UK; ${ }^{9}$ Bristol Breast Care Centre, North Bristol NHS Trust, Southmead Road, Bristol BS10 5NB, UK and ${ }^{10}$ Linda McCartney Centre, Royal Liverpool and Broadgreen University Hospital, Prescot Street, Liverpool L7 8XP, UK

Correspondence: Shelley Potter (shelley.potter@bristol.ac.uk)

These authors contributed equally: Shelley Potter, Chris Holcombe

Members of the iBRA-2 Steering Group and Breast Reconstruction Research Collaborative are PUBMED citable collaborators and are listed below Acknowledgements section.
}

Received: 16 September 2018 Revised: 18 February 2019 Accepted: 18 February 2019

Published online: 29 March 2019 
significant heterogeneity and these results cannot be relied upon. Two large population-based studies, however have recently reported delays to the start of chemotherapy in the patients undergoing IBR. One study did not differentiate between types of breast reconstruction ${ }^{7}$ and the second used patients undergoing breast conserving surgery as a control group and demonstrated delays in patients undergoing mastectomy without reconstruction as well as those undergoing immediate autologous reconstruction procedures ${ }^{8}$ making these findings difficult to interpret.

High-quality evidence regarding the impact of IBR on the delivery of adjuvant therapy compared with mastectomy alone is therefore lacking. Randomised trials (RCTs) provide the best evidence of treatment effect but are inappropriate in this context. A large-scale prospective cohort study is therefore required to generate high-quality data to allow patients and surgeons to make more informed decisions about potential treatment options. The trainee research collaborative model has recently emerged as a time and cost-effective method for delivering large-scale prospective studies in reconstructive breast surgery. ${ }^{12}$ This network of breast and plastic surgeons was utilised to deliver the iBRA-2 study to determine the impact of IBR on the delivery of adjuvant treatment. ${ }^{13}$

\section{METHODS}

Study design and participants

A prospective multicentre cohort study was used to determine whether IBR influenced time to delivery of adjuvant therapy compared to mastectomy alone.

All breast or plastic surgical units performing mastectomy with or without IBR were invited to participate through the UK Trainee Collaborative Research Network (the Mammary Fold Academic and Research Collaborative and the Reconstructive Surgery Trials Network) and the UK professional associations (Association of Breast Surgery [ABS] and the British Association of Plastic Reconstructive and Aesthetic Surgeons [BAPRAS]).

Consecutive women aged 18 or over undergoing mastectomy with or without IBR using any technique for invasive or preinvasive (ductal carcinoma in situ, DCIS) breast cancer with curative intent at participating centres between 1st July and 31 st December 2016 were recruited to the study. Excluded were patients undergoing risk-reducing surgery (without a therapeutic mastectomy for breast cancer), partial mastectomy including wide local excision with volume replacement (latissimus dorsi miniflaps; lateral intercostal perforator (LICAP) or thoracodorsal artery perforator (TDAP) flaps) or displacement techniques (therapeutic mammaplasty), and those with distant metastatic disease.

This study was classified as service evaluation by the UK National Health Service Research Authority Decision Tool (http:// www.hra-decisiontools.org.uk/research/index.html), hence individual patient consent was not required. Each participating centre registered the study and obtained local clinical governance approvals before commencing patient recruitment. The study protocol was published in $2016 .^{13}$

\section{Procedures}

Patients were identified prospectively from clinics, multidisciplinary team (MDT) meetings and operating theatre lists. Simple demographic, comorbidity, operative and oncology data were collected for each participant. Decisions regarding the recommendation for adjuvant treatment were identified from the postoperative MDT meeting.

For patients in whom adjuvant therapy was recommended, data were collected on whether the offer was accepted and in patients electing to receive adjuvant therapy, date of the first treatment was recorded.

Data regarding post-operative complications were collected prospectively until the patient commenced adjuvant therapy or it was decided that adjuvant therapy would be omitted due to postoperative complications. Preliminary work suggested that adjuvant therapy was unlikely to commence earlier than 6 weeks postoperatively. Data collection in patients not requiring adjuvant treatment therefore continued from the last definitive cancer surgery until 6 weeks following surgery either by clinical assessment or note-review in those not attending for follow-up.

The REDCap electronic data-capture system ${ }^{14}$ (http://www. projectredcap.org/) was used in data collection.

The study processes were piloted over a 4-week period to ensure the feasibility of the study and to refine the case report forms before commencing national recruitment.

For the purposes of the analysis, patients were categorised into four groups according to the most complex procedure received as: (i) mastectomy only without reconstruction; (ii) mastectomy and IBR with implant-only techniques; (iii) mastectomy and IBR with pedicled flaps and (iv) mastectomy and IBR with free-flap techniques. Implant-based procedures included any reconstruction in which only expanders/implants were used to reconstruct the breast. This included one or two-stage procedures with or without biological (e.g. acellular dermal matrix) or synthetic (e.g. titanium-coated polypropylene) mesh irrespective of whether the implant/expander was placed in a pre- or subpectoral position. Pedicled flap procedures included any pedicled flap used to reconstruct the breast with or without an implant/expander and included latissimus dorsi (LD) and transverse rectus abdominus myocutaneous (TRAM) flaps. Free-flap procedures included any technique in which a microvascular free-flap was used for IBR and included deep inferior epigastric perforator (DIEP), superficial inferior epigastric perforator (SIEA), superior and inferior gluteal artery perforator (SGAP and IGAP) and transverse upper gracillis, (TUG) procedures.

\section{Outcome measures}

The primary outcome was time in days from last definitive cancer surgery to the first adjuvant treatment. The last definitive cancer surgery included any additional procedures recommended by the MDT for oncological reasons (e.g. axillary clearance) but did not include any surgery for post-operative complications (e.g. debridement of skin-flap necrosis). First adjuvant therapy was defined as the first dose of chemotherapy or the first fraction of radiotherapy. Time to endocrine therapy was not included. In patients for whom more than one modality of adjuvant treatment was recommended, only the start date for the first adjuvant therapy was recorded. Significant treatment delays to (i) chemotherapy and (ii) radiotherapy were defined based on the best available evidence ${ }^{7,8,10}$ as delays of $>90$ days for chemotherapy ${ }^{7,8}$ and $>8$ weeks for radiotherapy. ${ }^{10}$

Secondary outcomes included post-operative complications, readmission to hospital following discharge and unplanned reoperation for complications within 6 weeks of the last definitive cancer surgery or prior to the start of adjuvant therapy. All complications were defined a priori. Major complications were defined as any complication requiring re-admission or reoperation. Minor complications were defined as those managed conservatively. ${ }^{13}$

\section{Quality assurance}

For quality assurance (QA) purposes, the principal investigator at each participating site was asked to independently validate $5-10 \%$ of the submitted data for each unit and to check complete case ascertainment. If concordance between the data entered on REDCap and that independently validated was $<90 \%$, the unit's data were excluded from the analysis consistent with the QA procedure used in other collaborative projects. ${ }^{13}$

\section{Statistical analysis}

Descriptive summary statistics were calculated for each variable for the cohort overall and split by operative procedure. Categorical 
Table 1. Demographics of participants in the iBRA-2 study by procedure type

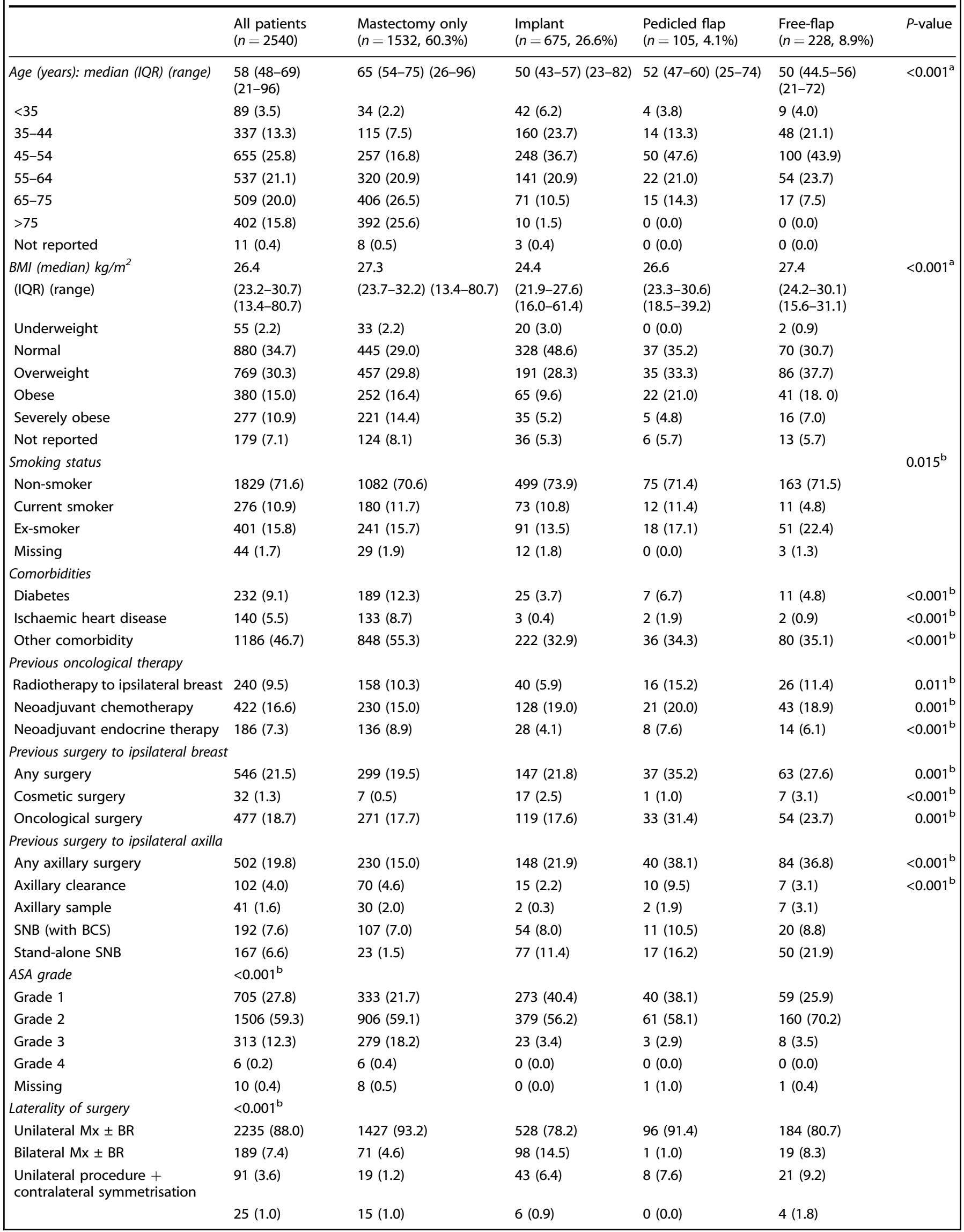




\begin{tabular}{|c|c|c|c|c|c|c|}
\hline & $\begin{array}{l}\text { All patients } \\
(n=2540)\end{array}$ & $\begin{array}{l}\text { Mastectomy only } \\
(n=1532,60.3 \%)\end{array}$ & $\begin{array}{l}\text { Implant } \\
(n=675,26.6 \%)\end{array}$ & $\begin{array}{l}\text { Pedicled flap } \\
(n=105,4.1 \%)\end{array}$ & $\begin{array}{l}\text { Free-flap } \\
(n=228,8.9 \%)\end{array}$ & $P$-value \\
\hline \multicolumn{7}{|l|}{$\begin{array}{l}\text { Unilateral procedure }+ \\
\text { contralateral oncological } \\
\text { procedure }\end{array}$} \\
\hline $\begin{array}{l}\text { Indication for bilateral surgery } \\
(n=305)\end{array}$ & $<0.001^{\mathrm{b}}$ & & & & & \\
\hline $\begin{array}{l}\text { Unilateral malignancy/ } \\
\text { contralateral risk reduction }\end{array}$ & $116(38.0)$ & $35(33.3)$ & $66(44.9)$ & $2(22.2)$ & $13(29.6)$ & \\
\hline $\begin{array}{l}\text { Unilateral malignancy/ } \\
\text { contralateral symmetrisation }\end{array}$ & $93(30.5)$ & $20(19.1)$ & $42(28.6)$ & 7 (77.8) & $24(54.6)$ & \\
\hline Unilateral malignancy/other & $14(4.6)$ & $11(10.5)$ & $3(2.0)$ & $0(0.0)$ & $0(0.0)$ & \\
\hline
\end{tabular}

data were summarised by counts and percentages. Continuous data were summarised by median, interquartile range (IQR) and range. Procedure groups were compared using appropriate nonparametric statistics. Complications and oncological outcomes were summarised by procedure and by patient.

Univariable and multivariable logistic regression analysis was used to explore clinico-pathological variables hypothesised to be associated with the development of (i) any complication and (ii) major complications, as these were considered most likely to impact on time to adjuvant therapy. Variables of interest were defined a priori based on the literature and expert opinion and included patient and procedure-related variables, namely age, smoking, body mass index (BMI), diabetes, ischaemic heart disease (IHD), other comorbidities, previous surgery and/or radiotherapy to the ipsilateral breast; neoadjuvant chemotherapy (NAC), American Society of Anesthesiologists (ASA) grade, unilateral vs bilateral surgery, type of axillary surgery (none, sentinel node biopsy [SNB] or axillary node clearance [ANC]) and procedure type (mastectomy, implant-based, pedicled or free-flap reconstruction).

Time from surgery to first adjuvant therapy was calculated for all patients and for those undergoing (i) chemotherapy and (ii) radiotherapy as their first adjuvant treatment separately in each procedure group, with adjuvant therapy as the event. This analysis was repeated stratifying by whether the patient had no, minor, or major complications. Kaplan-Meier analyses, univariable and multivariable Cox survival models of time to first adjuvant therapy and time to (i) chemotherapy and (ii) radiotherapy separately split by procedure type were created, including patient age, BMI, diabetes, IHD, other comorbidities, smoking, ASA grade, unilateral vs bilateral surgery, procedure type and the presence of complications (none, minor or major) as variables of interest, clustered by centre. The Kaplan-Meier graphs of time to adjuvant therapy were curtailed at 150 days, when only 10 patients remain in follow-up, to better focus on the majority of patients.

STATA 15 (STATA, Inc., Texas) was used for all analyses.

\section{RESULTS}

In total, 2652 patients were recruited to the study from 76 centres across the UK $(n=66)$, Europe $(n=9)$ and North Africa $(n=1)$. Of these, 112 (4.4\%) were excluded; $19(0.7 \%)$ had surgery outside of the study period; $55(2.1 \%)$ had risk-reducing surgery only; 6 $(0.2 \%)$ did not undergo a mastectomy and $24(0.9 \%)$ had incorrect or important missing data (e.g. operation date or procedure type). Eight $(0.3 \%)$ patients had 'other' forms of reconstruction. These could not be appropriately categorised, hence were excluded.
2540 patients were therefore included in the analysis. Of these, 1008 (39.7\%) underwent IBR with implant-based $(n=675)$, pedicled flaps $(n=105)$ or free-flap $(n=228)$ techniques.

Patient demographics

Patient demographics are summarised in Table 1. Women undergoing IBR were younger and had fewer comorbidities than patients undergoing mastectomy only. More patients undergoing IBR received NAC than those undergoing simple mastectomy and patients undergoing IBR were more likely to have undergone an up-front SNB before their reconstruction, particularly if they were undergoing tissue-based procedures. Bilateral surgery for risk reduction or symmetry was more common in patients undergoing implant-based or free-flap reconstruction (Table 1).

Post-operative complications

The 2540 patients underwent 2732 procedures including 773 implant-based reconstructions (157 subpectoral expanders; 410 subpectoral reconstructions with biological or synthetic mesh; 105 dermal-sling procedures and 98 prepectoral reconstructions), 106 pedicled flaps (62 autologous LD, 39 LD with implant, 2 pedicled TRAM and 2 other) and 247 free-flap procedures (219 DIEPs, 16 free TRAMs, 4 SIEA, 7 TUG flaps and 1 other). Details of complications by procedure are summarised in supplementary table 1.

Overall, $929(36.6 \%)$ of patients in the study experienced at least one post-operative complication (Table 2). Univariable analysis identified age, BMI, IHD, diabetes, having other comorbidities, smoking, ASA grade and undergoing an ANC but not IBR as risk factors associated with developing a post-operative complication (Table 2). Age, BMI, having other comorbidities, smoking and undergoing an ANC remained strongly associated with postoperative complications in the multivariable model, whereas undergoing bilateral surgery and free-flap reconstruction were also identified as independent risk factors for complications in the multivariable analysis.

Major complications which required re-admission to hospital or further surgery (Table 2) were experienced by $221(8.7 \%)$ of patients. Implant-based and free-flap reconstruction, age, BMI, smoking and bilateral surgery were associated with major complications in the univariable analysis. All of these variables except for age, remained strongly associated with major complications in the multivariable model but implant-based (adjusted odds ratio [aOR] 4.34, 95\% confidence interval [Cl] 2.35-7.99) and free-flap reconstruction (aOR 4.88, 95\% Cl 2.63-9.04) were the strongest predictors for major complications in this analysis (Table 2). 


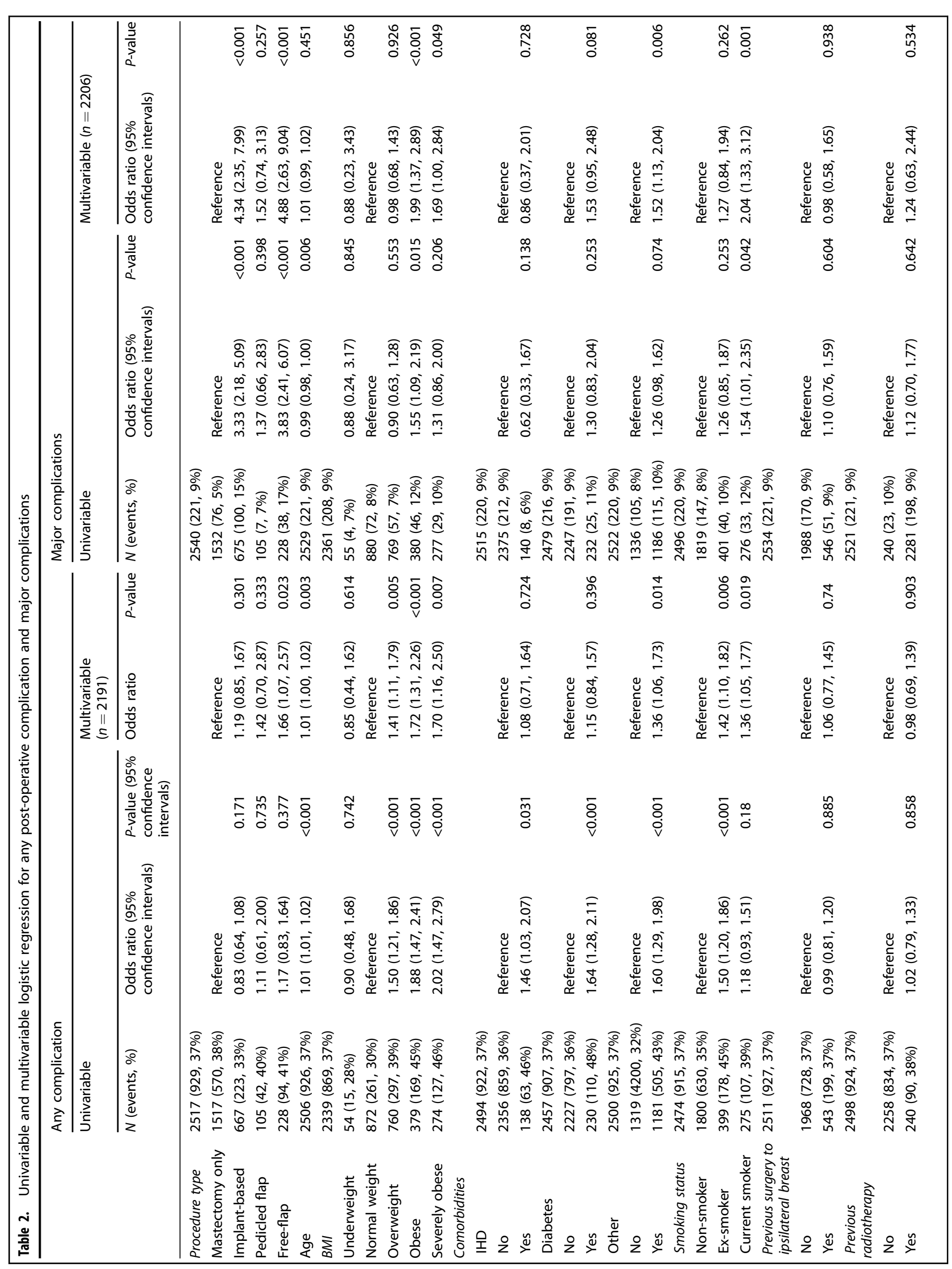




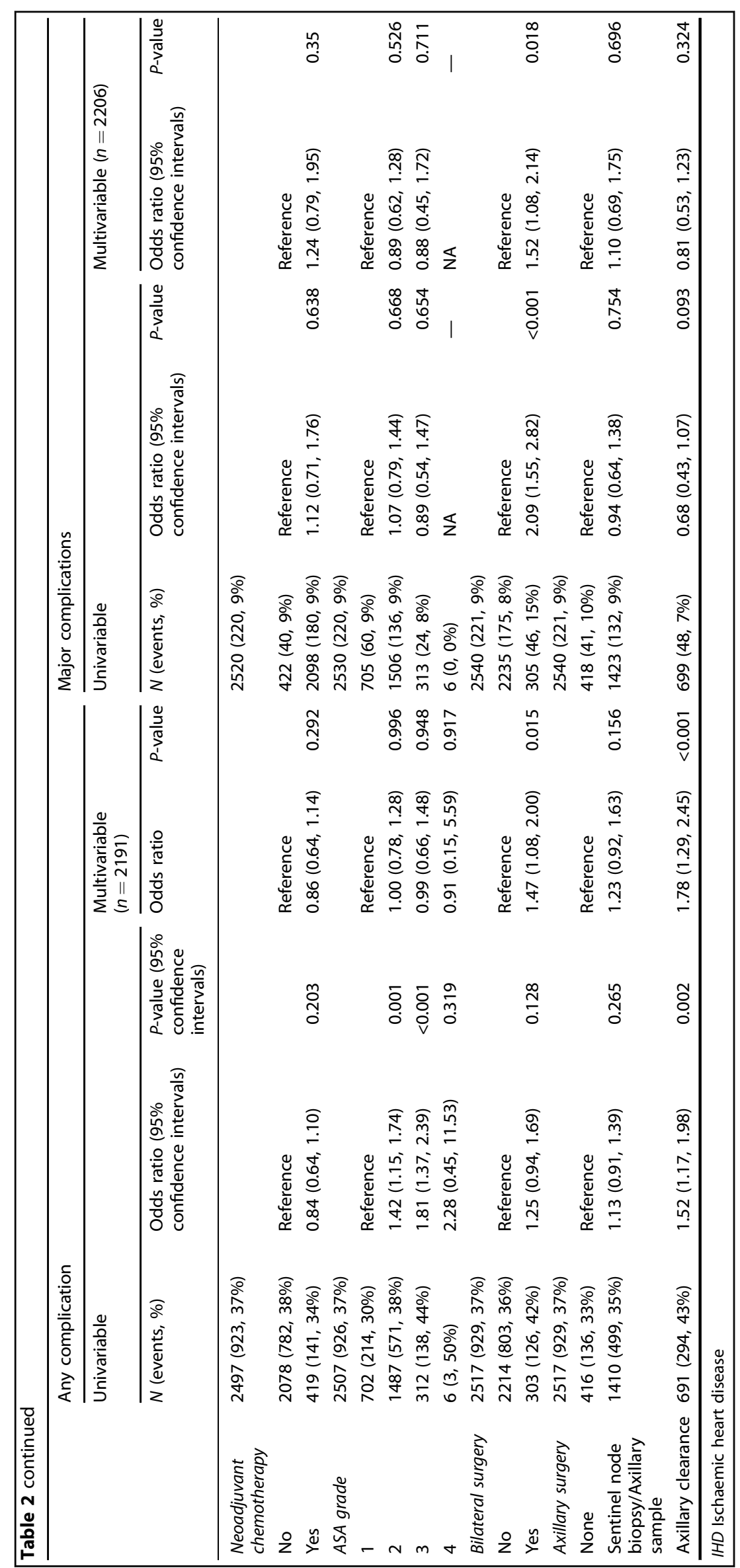


Adjuvant treatment recommendations and time to adjuvant therapy

Table 3 summarises the post-operative pathology for the 2607 mastectomies performed for oncological indications. IBR was more likely to be performed following mastectomy for extensive DCIS or multifocal disease and in node-negative patients than simple mastectomy resulting in fewer patients in the IBR group requiring adjuvant chemotherapy or radiotherapy.

Overall, 1235 (48.6\%) patients were offered and accepted adjuvant treatment (Table 4). Time to adjuvant treatment differed between the groups, with those undergoing free-flap procedures having longer time to adjuvant therapy than those undergoing

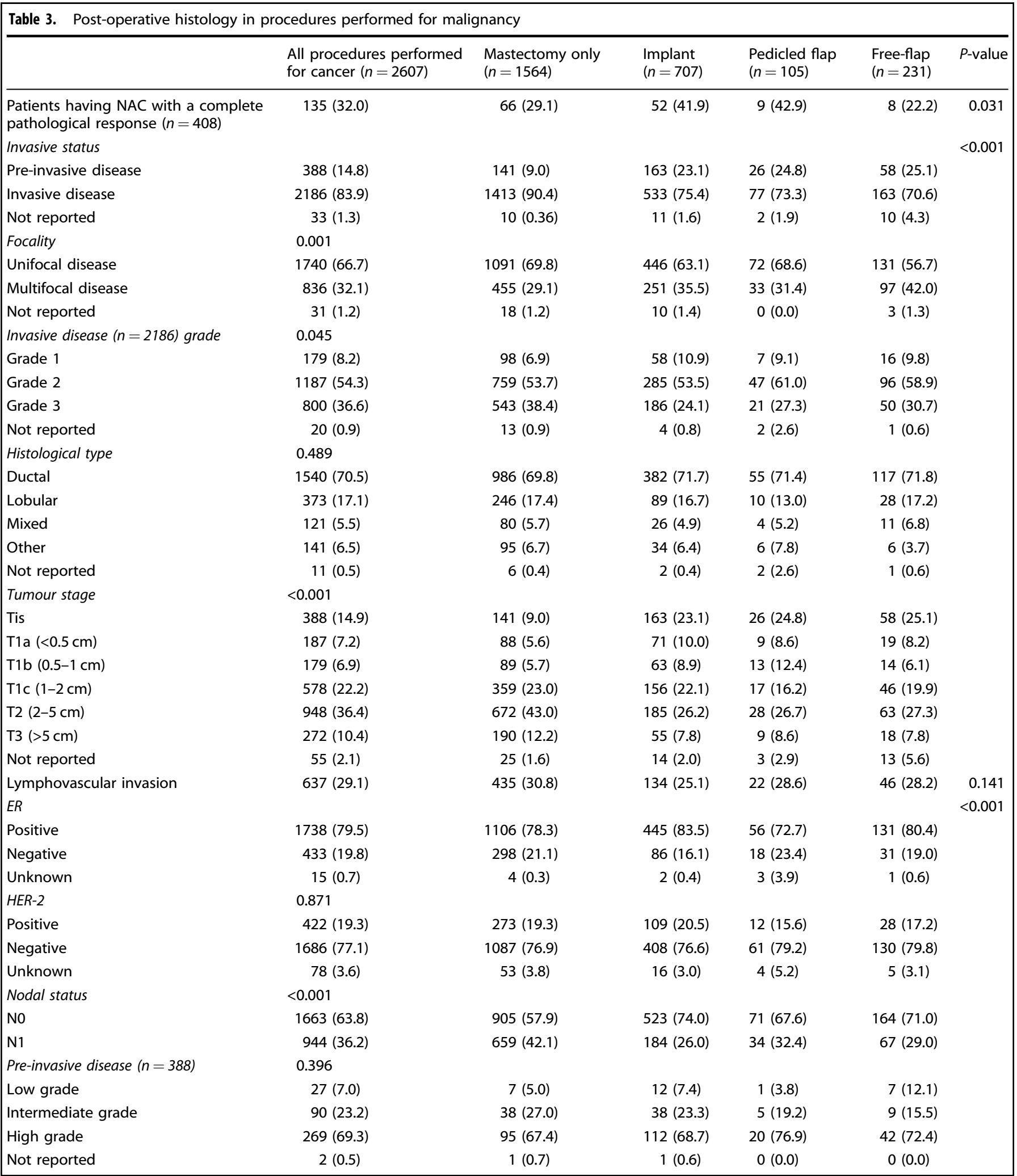


Table 4. Multidisciplinary team (MDT) decision-making for adjuvant therapy

\begin{tabular}{|c|c|c|c|c|c|c|}
\hline MDT decision-making per patient & $\begin{array}{l}\text { All patients } \\
(n=2540)\end{array}$ & $\begin{array}{l}\text { Mastectomy only } \\
(n=1532)\end{array}$ & $\begin{array}{l}\text { Implant } \\
(n=675)\end{array}$ & $\begin{array}{l}\text { Pedicled flap } \\
(n=105)\end{array}$ & $\begin{array}{l}\text { Free-flap } \\
(n=228)\end{array}$ & $P$-value \\
\hline \multicolumn{7}{|l|}{ Chemotherapy } \\
\hline For discussion with patient & $188(7.4)$ & $138(9.0)$ & $36(5.3)$ & $5(4.8)$ & $9(3.9)$ & \\
\hline For oncotype DX testing & $181(7.1)$ & $95(6.2)$ & $64(9.5)$ & $5(4.8)$ & $17(7.5)$ & \\
\hline Not recommended by MDT & $1509(59.4)$ & $872(56.9)$ & $415(61.5)$ & $67(63.8)$ & $155(68.0)$ & \\
\hline \multicolumn{7}{|l|}{ Radiotherapy } \\
\hline Recommended by MDT & $909(35.7)$ & $614(40.1)$ & $198(29.3)$ & $35(33.3)$ & $62(27.2)$ & $<0.001$ \\
\hline For discussion with patient & $125(4.9)$ & $86(5.6)$ & $19(2.8)$ & $1(1.0)$ & $19(8.3)$ & \\
\hline Not recommended by MDT & $1492(58.7)$ & $828(54.0)$ & $449(66.5)$ & $69(65.7)$ & $146(64.0)$ & \\
\hline Not reported & $14(0.5)$ & $4(0.3)$ & $9(1.3)$ & $0(0.0)$ & $1(0.4)$ & \\
\hline $\begin{array}{l}\text { Patient accepts adjuvant treatment (either } \\
\text { chemotherapy or radiotherapy or both) }\end{array}$ & $1235(48.6)$ & $804(52.5)$ & $288(42.7)$ & $50(47.6)$ & $93(40.8)$ & $<0.001$ \\
\hline Chemotherapy as $1 \mathrm{st}$ adjuvant treatment & $627(55.4)$ & $409(55.4)$ & $147(56.5)$ & $25(52.1)$ & $46(54.1)$ & 0.939 \\
\hline $\begin{array}{l}\text { Time from last oncological procedure to } \\
\text { 1st chemotherapy (days) median (IQR) }\end{array}$ & $47(37-59)$ & $47(37-59)$ & $46(35-57)$ & $46(39-58)$ & $57(41-70)$ & 0.063 \\
\hline $\begin{array}{l}\text { Reported delays of }>90 \text { days of planned } \\
\text { chemotherapy }(n=637)\end{array}$ & $31(4.9)$ & $21(5.1)$ & $4(2.7)$ & $3(11.5)$ & $3(6.5)$ & 0.228 \\
\hline Radiotherapy as 1 st adjuvant treatment & $504(44.6)$ & $329(44.6)$ & $113(43.5)$ & $23(47.9)$ & $39(45.9)$ & 0.939 \\
\hline $\begin{array}{l}\text { Time from last oncological procedure to } \\
\text { 1st radiotherapy (days) median (IQR) }\end{array}$ & $60(48-73)$ & $59(48-73)$ & $60(45-68)$ & $63(53-85)$ & $62(50-76)$ & 0.248 \\
\hline $\begin{array}{l}\text { Reported delays of }>56 \text { days ( } 8 \text { weeks) of } \\
\text { planned radiotherapy }(n=616)\end{array}$ & $389(63.2)$ & $258(62.8)$ & $83(61.9)$ & $23(79.3)$ & $25(59.5)$ & 0.308 \\
\hline
\end{tabular}

mastectomy only, adjusted hazard ratio (aHR) $0.84(95 \% \mathrm{Cl}$ 0.71-0.99) (Table 5, Fig. 1a). The absolute differences between the median time to adjuvant treatment across the groups, however, were small; 52 (IQR 41-66) days for mastectomy only vs 57 (IQR 46-72) days for free-flap reconstruction (Table 5). The development of complications (Fig. 1b) and obesity were also associated with longer time to adjuvant therapy (Table 5). Median time to first chemotherapy was 47 days, (IQR 37-59). There were no significant differences in median time to chemotherapy or in the proportions of patients experiencing delays of greater that 90 days between the treatment groups (Table 4) but free-flap reconstruction (aHR 0.79, [95\% Cl 0.65-0.96]), major complications (aHR 0.72, [95\% Cl 0.54-0.94]) and obesity (aHR 0.75, [95\% Cl 0.57-0.99]) were associated with having longer time to chemotherapy in the multivariable model (Supplementary table 2). Median time to first fraction of radiotherapy was 60 days (IQR 48-73) with no differences in either the median time to radiotherapy or the proportion of patients experiencing significant treatment delays, defined as $>8$ weeks, between procedure types (Table 4). Major complications (aHR 0.70, [95\% Cl 0.53-0.93]) and smoking (aHR 0.73, [95\% Cl 0.57-0.94]) were associated with longer time to adjuvant radiotherapy in the multivariable model with older patients and those who had received neoadjuvant chemotherapy proceeding to radiotherapy more rapidly than other patient groups (Supplementary table 3).

Time to first adjuvant therapy $(P<0.001)$, time to chemotherapy $(P<0.001)$ and time to radiotherapy $(P=0.026)$, however all differed by whether the patient had no, minor or major complications, with an increasing trend seen across the three groups (no complications 50 days [IQR 39-63]; minor complications 56 days [IQR 42.5-69]; major complications 57 days [IQR 46-73], Supplementary table 4). Furthermore, patients experiencing complications were significantly more likely to experience significant treatment delays, defined as delays of $>90$ days for chemotherapy $(n=14,3.6 \%$ of patients with no complications vs $n=7,13 \%$ of patients with major complications; $P=0.011)$ and $>8$ weeks for radiotherapy $(n=222,58.7 \%$ of patients with no complications vs $n=29,70.7 \%$ of patients with major complications; $P=0.016$, Supplementary table 4) than those whose procedures were uncomplicated.

\section{DISCUSSION}

Although free-flap reconstruction was associated with a longer time to adjuvant therapy than other procedure types, the absolute differences in time to treatment between the surgical groups is small. This study therefore suggests that IBR does not result in clinically significant delays in the delivery of adjuvant therapy compared to mastectomy alone. Complications, especially those requiring re-admission or further surgery however, are important and patients developing problems, irrespective of the procedure performed, were more likely to experience significant delays to both chemotherapy and radiotherapy in this analysis. The apparent paradox of no treatment delay despite the higher rate of major post-operative complications in the IBR group can be explained by careful patient selection for reconstructive surgery. Patients undergoing IBR were significantly younger and fitter, with fewer 'risk factors' for complications than patients undergoing mastectomy only and were less likely to require adjuvant treatment than the mastectomy only group. This is because IBR was more likely to be performed following mastectomy for extensive DCIS than for high-risk invasive disease with upfront axillary staging used to determine the likelihood that patients would require adjuvant treatment before their reconstructive 
Table 5. Cox univariable and multivariable survival analyses for time to adjuvant treatment

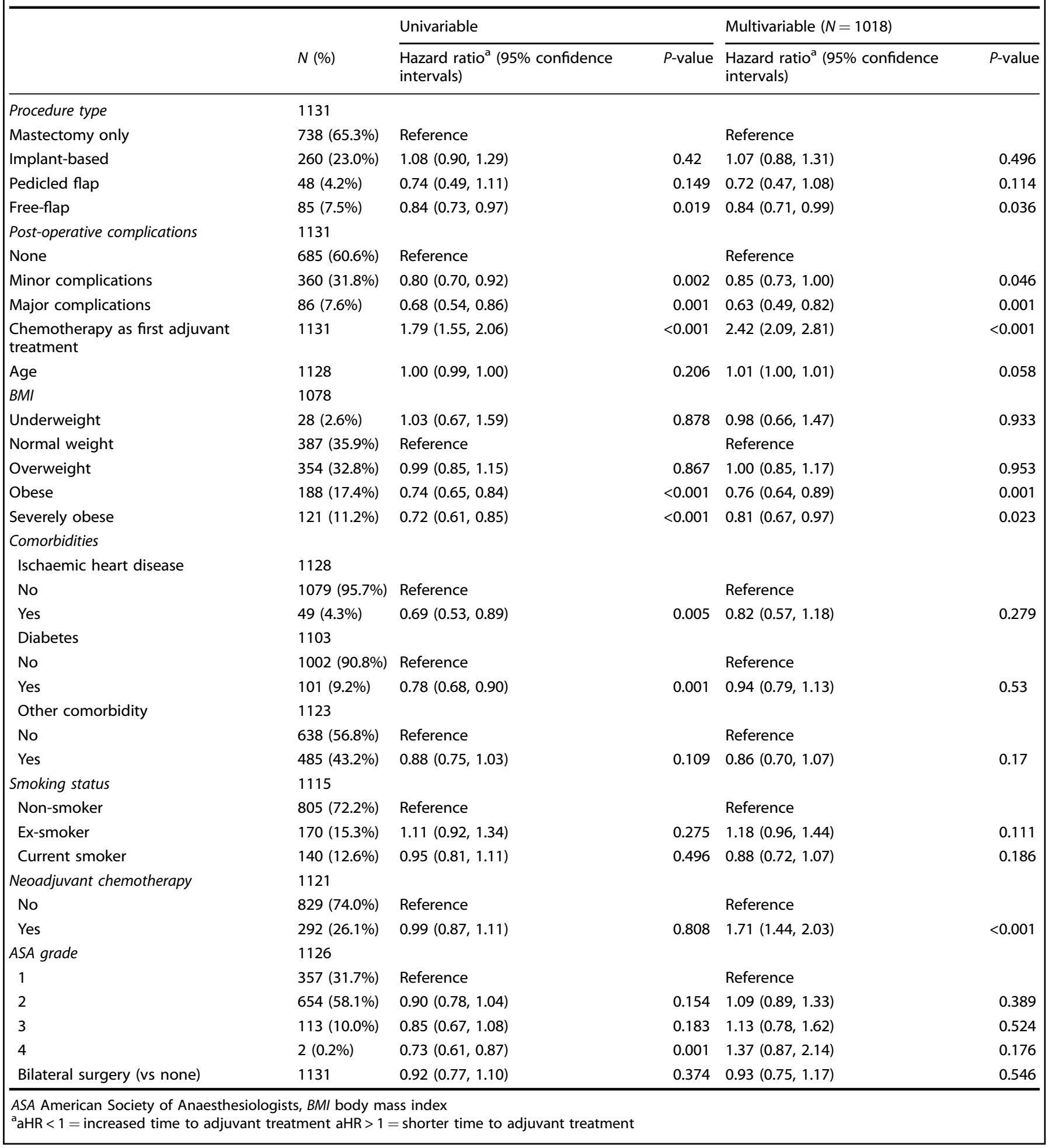

procedure. This suggests that surgeons are cautious in offering IBR to patients likely to require adjuvant treatment. ${ }^{15}$ These concerns may reflect the impact of radiotherapy on the cosmetic outcome of reconstruction, ${ }^{16}$ but may also highlight anxiety about potential delays to adjuvant treatment with surgeons only opting to perform IBR in patients considered low risk. This study provides much-needed evidence to suggest that IBR does not lead to clinically significant delays in carefully selected low risk patient groups but does highlight that major complications can result in significant treatment delays. This study therefore strongly supports the need for careful patient selection to minimise complications and careful communication of the risks of postoperative problems and the potential oncological implication of complications on treatment delays with patients considering surgery. The higher risk of complications in patients undergoing bilateral surgery will particularly inform discussions with patients 


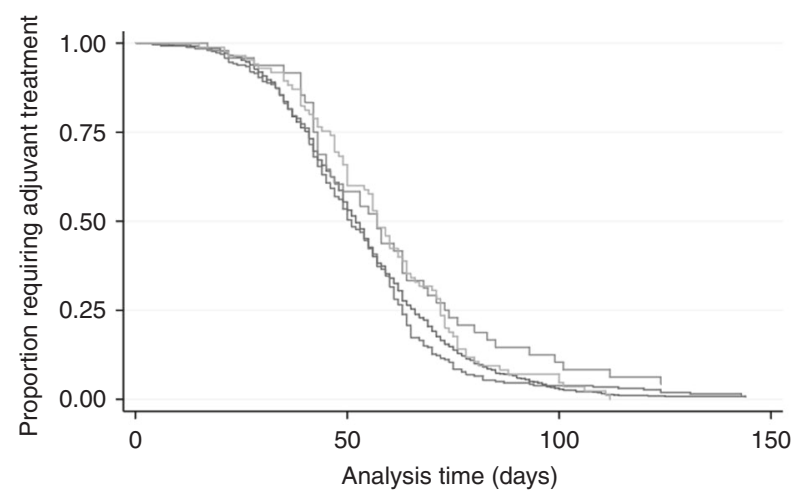

Mastectomy only $\longrightarrow$ Implant-based

$\begin{array}{lcccc}\text { Number remaining: } & 0 \text { days } & 50 \text { days } & 100 \text { days } & 150 \text { days } \\ \text { Mastectomy only } & 738 & 409 & 23 & 5 \\ \text { Implant-based } & 260 & 139 & 10 & 3 \\ \text { Pedicled flap } & 48 & 28 & 5 & 2 \\ \text { Free flap } & 85 & 56 & 6 & 0\end{array}$

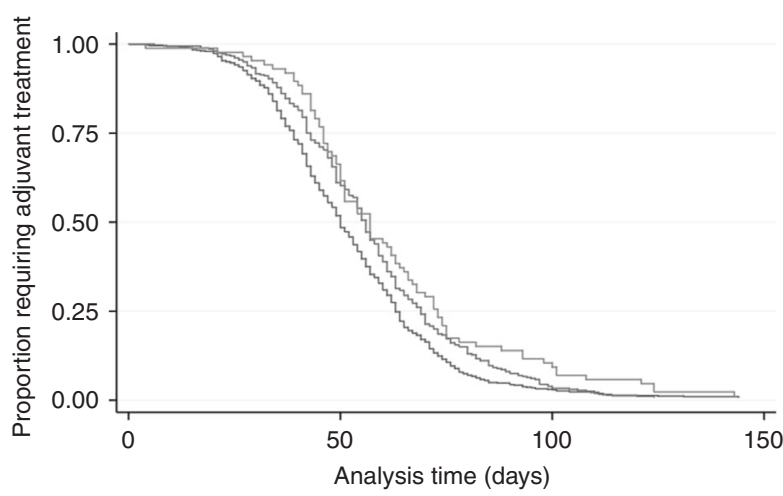

\begin{tabular}{|c|c|c|c|c|}
\hline & \multicolumn{2}{|c|}{$\begin{array}{l}\text { None } \\
\text { Major complications }\end{array}$} & \multicolumn{2}{|c|}{ - Minor complications } \\
\hline & 0 days & 50 days & 100 days & 150 days \\
\hline No complications & 685 & 355 & 21 & 6 \\
\hline Minor complications & 360 & 220 & 14 & 3 \\
\hline Major complications & 86 & 57 & 9 & 1 \\
\hline
\end{tabular}

86

Fig. 1 Kaplan-Meier analyses for time from last oncological surgery to first adjuvant treatment by a procedure type (left), b whether or not the patient developed post-operative complications (right)

wishing to undergo simultaneous contralateral risk-reducing mastectomy and gives a sound rationale for delaying such surgery if adjuvant therapy is anticipated, particularly in implantbased reconstruction.

The findings of this study are consistent with other work suggesting that post-operative complications, rather than procedure type, are the main predictor of adjuvant treatment delays. ${ }^{8}$ This focuses attention on the need to reduce complications to improve outcomes for patients and is particularly relevant as reconstruction rates are increasing. ${ }^{17}$ Despite more procedures being performed, however, complications rates appear to be rising with re-operation for complications more than double that seen in the UK National Mastectomy and Breast Reconstruction Audit (NMBRA)..$^{5}$ This is a cause for concern as complications not only delay delivery of adjuvant treatments and but may also adversely impact long-term oncological outcomes by promoting a systemic inflammatory response. ${ }^{18}$ Implant-based procedures are now the most commonly-performed technique ${ }^{19,20}$ and although data from the NMBRA ${ }^{5}$ and the National Surgical Quality Improvement Program ${ }^{21}$ suggest implant reconstruction may be associated with fewer complications than other techniques, this study suggests that complications following implant-based and autologous reconstruction are broadly comparable. Reasons for this require further evaluation but may reflect the recent adoption of singlestage direct-to-implant mesh-assisted reconstruction in the $\mathrm{UK}^{22}$ which may be associated with higher complication rates than the traditional two-stage procedures ${ }^{23}$ favoured in the US. ${ }^{20}$ Risk factors for complications, including smoking and high BMI are consistent with those previously reported ${ }^{24,25}$ and highlight the importance of careful patient selection if post-operative problems are to be avoided.

This is the first large prospective multicentre study to explore the impact of IBR on time to adjuvant therapy, but it has limitations. Firstly, this is an observational study and risk of bias must be considered. Consecutive patients undergoing mastectomy were recruited from participating centres but there were baseline differences in the treatment groups. Although it was possible to adjust for confounding factors such as age, BMl, smoking and ASA grade in the regression analyses, it is acknowledged that it is not possible to identify and control for all potential confounders which may have impacted the results. The study included patients from 76 centres across the UK and Europe and it is the largest study of its kind, but it is possible that participating units differed from those not taking part. However, this is unlikely, as almost half of all the breast and plastic surgical units in the UK elected to participate. A further consideration is that by only reporting delay to initiation of treatment, this study may underestimate both the overall complication rate of IBR and the true impact of reconstruction on the delivery of adjuvant therapy. This is particularly relevant for patients having implant reconstruction who may develop infection while receiving chemotherapy requiring treatment to be modified or stopped completely and the implant removed. Following patients during adjuvant treatment was not feasible with the trainee collaborative study design, but new collaborations with oncology trainees will allow these issues to be addressed in the future. Finally, this short-term study does not allow the long-term oncological impact of post-operative complications or any delays in the delivery of adjuvant therapy to be assessed. A data-linkage study to explore long-term oncological outcomes at 5 and 10 years is planned, allowing these important questions to be addressed. Therefore, although it is not possible to establish causality with an observational study design, RCTs in this setting are not possible and the iBRA-2 study provides much-needed evidence to support decision-making for IBR when adjuvant treatments may be needed.

The development of post-operative complications rather than the type of procedure performed has emerged as the key determinant of delays to the delivery adjuvant therapy in this study. Immediate implant-based and free-flap reconstructions, however, are associated with significantly higher rates of major complications than mastectomy alone and this is an important finding that should be fully discussed with patients considering reconstructive surgery. Avoiding IBR in high-risk patients including smokers and those with a high BMI and not performing unnecessary bilateral surgery may represent a simple strategy for reducing post-operative problems but this approach needs balanced against patients' desire for IBR. Accurate and balanced communication of risks and benefits is a vital part of shared decision-making, ${ }^{26}$ and this study provides further evidence to inform this discussion. Major complications, irrespective of the procedure performed, result in delays to adjuvant treatment, hence strategies to minimise complications are needed for all patients undergoing breast cancer surgery to improve oncological outcomes, $^{18}$ quality-of-life ${ }^{27}$ and minimise the overall cost of 
care. $^{28}$ Standardising care may be one strategy by which outcomes may be improved and standardisation is the focus of the UK 'Getting it Right First Time' initiative. http:// gettingitrightfirsttime.co.uk/surgical-specialty/breast-surgery/. Other strategies include altering treatment sequencing and routinely using neoadjuvant rather than adjuvant chemotherapy in patients electing to undergo IBR. This approach is safe, and these data show that those having neoadjuvant therapy start their adjuvant therapy sooner. It may also allow patients to address modifiable risk factors such as obesity or smoking before surgery although it is appreciated that these changes may be challenging. Increased use of neoadjuvant endocrine therapy may also have utility in high-risk groups. Neoadjuvant radiotherapy is a novel approach, which may provide an alternative treatment pathway in patients in whom radiotherapy is likely to be required. ${ }^{29}$ More accurately determining which patients may benefit from adjuvant therapy before the start of their breast cancer treatment, however may be the optimal solution and work to develop a more personalised approach using molecular markers and gene signatures is likely to reduce the number of future patients in whom adjuvant treatment may be indicated. ${ }^{30,31}$

IBR does not delay the delivery of adjuvant therapy, but implant-based and free-flap reconstructions are associated with higher rates of post-operative complications which are associated with treatment delays. Careful patient selection combined with accurate communication of risk are therefore vital if patients are to make fully informed decision about IBR when adjuvant therapy is likely to be needed. Further strategies to minimise the risk of complications such as increased use of neoadjuvant treatment may also be beneficial in this group. This study provides important information about the risk and impact of complications in IBR to help patients and surgeons make more informed decisions about their treatment options.

\section{ACKNOWLEDGEMENTS}

We would like to thank the following for their contributions to the study. This work received no specific grant from any funding agency in the public, commercial or notfor-profit sectors. Shelley Potter is an NIHR Clinician Scientist (CS-2016-16-019). Tim Rattay has received support from the NIHR through a Doctoral Research Fellowship (DRF-2014-07-079) and Academic Clinical Lectureship. This work was undertaken with the support of the NIHR Biomedical Research Centre at University Hospitals Bristol NHS Foundation Trust and the University of Bristol. The views expressed in this publication are those of the authors and not necessarily those of the NHS, the National Institute for Health Research or the Department of Health and Social Care.

\section{MEMBERS OF THE IBRA-2 STEERING GROUP ARE (IN ALPHABETICAL ORDER)}

Nicola LP Barnes, Jane Blazeby, Elizabeth Conroy, Rajiv V Dave, Matthew Gardiner, Adrian Harnett, Chris Holcombe, Ciara O'Brien, Rachel O'Connell, Shelley Potter, Tim Rattay, Joanna Skillman, and Paula Williamson.

\section{LOCAL INVESTIGATORS (ALPHABETICALLY BY CENTRE) AND MEMBERS OF THE BREAST RECONSTRUCTION RESEARCH COLLABORATIVE ARE PUBMED CITABLE COLLABORATORS}

Aberdeen Royal Infirmary, NHS Grampian; Alain Curnier, Amir Tadros, Ivan Depasquale, Yazan A Masannat, Elizabeth Smyth, Mairi Fuller, Roger Bourne, Steven Heys, Ishrak Hamo. Addenbrookes Hospital, Cambridge Universities NHS Foundation Trust; Fatima Aloraifi, Laura Fopp, Radhika Bali, Sarah Bache, Sarah L Benyon, Michael S Irwin, Amit Agrawal, Charles M Malata. Airedale NHS Foundation Trust; Claire Murphy. Basildon and Thurrock Hospitals NHS Foundation Trust; Adam Misky, Dennis Wayne Chicken. Beaumont Hospital and the RCSI; Nassreen Abdullah, Arnold D K Hill. Belfast City Hospital, Belfast Health and Social Care Trust; Carolyn Cullinane, Gareth Irwin, Stuart A McIntosh, Sigi Refsum, Samantha Sloan, Peter Mallon. Betsi Cadwaladr University Health Board; Chiara Sirianni, llyas Khattak, Chiara Sirianni. Blackpool Teaching Hospitals NHS Foundation Trust; Geerthan Nagachandra, Pasupathy Kiruparan, Debasish Debanth. Breast Care Centre, North Bristol NHS Trust, Southmead Hospital, Bristol; Simon Davey, Terry-Ann Curran, Matilda Svenning, Sasirekha
Govindarajulu, Zenon Rayter, Rachel Ainsworth, Simon Cawthorn, Ajay Sahu, Sherif Wilson, Elena Prousskaia. Breast Unit Department. University of Naples "Federico II", Naples, Italy; Antonello Accurso, Nicola Rocco, Rosa Di Micco, Antonello Accurso, Gennaro Limite. Breast Unit Department S. Maria delle Grazie ASL Na 2 Pozzuoli; Raffaele Ceccarino, Raffaele Liccardo, Guido Coco. Broomfield Hospital, Mid Essex Hospital Services NHS Trust; Metin Nizamoglu, Mary Morgan, Venkat Ramakrishnan. Cannizzaro Hospital, Catania; Giuseppe Catanuto. Castle Hill Hospital, Hull and East Yorkshire Hospitals NHS Trust; Alex Wilkins, Penelope McManus, Peter Kneeshaw, Kartikae Grover, Tapan Mahapatra, Brendan Wooler, Bilal Elahi, Naila Ihsan. Charing Cross Hospital, Imperial College Healthcare NHS Trust; Alexandra Bucknor, Dimitris Reissis, Judith Hunter, Simon Wood, Navid Jallali, Francis P Henry, Liaquat S Verjee, Jason Lee. Chesterfield Royal Hospital NHS Foundation Trust; Shazia M Khan, Iman Azmy, Julia Massey, Ciaran Hollywood, Michael Oluwajana. Countess of Chester Hospital NHS Foundation Trust; Sonia Bathla, Joanna Seward, Claudia HardingMacKean. Darent Valley Hospital, Dartford and Gravesham NHS Trust; Risha Lane, Kothandaraman Murali, Bashishta Biswas, Pawel Trapszo, Seema Seetharam. Dorset County Hospital NHS Foundation Trust; Katy Kennedy, Louise Alder, Tomasz Graja. East Cheshire NHS Trust; Khalid Amin, Jalal Kokan, Chandeena Roshanlall. Edinburgh Breast Unit, Western General Hospital, NHS Lothian; Emma Gill, Dhananjay Kulkarni, JM Dixon, Oliver Young, Talha Saleem. Glasgow Royal Infirmary, NHS Greater Glasgow and Clyde; M Biddle, Marie Kearns, Eva Weiler-Mithoff, Ben Chew, Andy Malyon, John Scott, David McGill, lain Mackay. Glenfield Hospital, University Hospitals of Leicester; Salena Bains, Sara Barrows, Tim Rattay, Simon Pilgrim, Sheila Shokuhi, Kelly Lambert, Frances Kenny, Kalliope Valassiadou, Monika Kaushik, Jaroslaw Krupa, Dimitris Dragoumis. Good Hope Hospital, Heart of England NHS Foundation Trust; Quratul ain, Pavlos Lampropoulos, Sarah Moss, Haitham Khalil, Anwar Haq, Balapathiran Balasubramanian. Guy's and St Thomas' NHS Foundation Trust; Petros Charalampoudis, Hisham Hamed, Ashutosh Kothari, Tibor Kovacs, Michael Douek. Harrogate and District NHS Foundation Trust; Iftikhar Mehmood, Biswajit Ray, Matthew Adelekan. Homerton University Hospital NHS Foundation Trust; Laura Humphreys, Salim Tayeh, Christina Choy, Laila Parvanta. Istituto Oncologico Veneto, Padova, Italy; Silvia Michieletto, Tania Saibene. James Paget University Hospitals NHS Foundation Trust; James O'Brien, Sue Down, Sarah Downey, Jerome Pereira. Lincoln County Hospital, United Lincolnshire Hospitals NHS Trust; A S Sami, Anzors Gvaramadze, Jibril A Jibril, Dinesh Thekkinkattil. Llanelli Peony Breast Unit; S Udayasankar, Saira Khawaja, Yousef Shariaha, Simon Holt. Luton and Dunstable University Hospital; Ruth James, Hirah Rizki, Katharine Kirkpatrick, Duraisamy Ravichandran, Deepak Shrestha. Maidstone and Tunbridge Wells NHS Trust; Ellora Barua, Deepika Akolekar. Mid Cheshire Hospitals NHS Foundation Trust; Ahmed Hamad, Eleftheria Kleidi, Susan Hignett, Vanessa Pope, Salma Naseem. Milton Keynes University Hospital NHS Foundation Trust; Jennifer Isherwood, Rachel Soulsby, Amanda Taylor, Kian Chin. Morriston Hospital, Abertawe Bro Morgannwg University Health Board; Dai Nguyen. Musgrove Park Hospital, Taunton and Somerset NHS Foundation Trust; Francesca Guest, Amanda Thorne. Nevill Hall Hospital, Aneurin Bevan University Health Board; Valentina Lefemine. Norfolk and Norwich University Hospitals NHS Foundation Trust; Chris Kirchhoff, Declan C Murphy, Michelle Lo, Ruth Harcourt, Simon J Pain, Maged I Hussien, Katalin Zechmeister, E.M. Sassoon, Andrea Figus, Richard M Haywood, Rozina Ali, Susanna Alexander, Adrian Harnett, Konstantinos Geropantas, Daniel Epurescu. North Middlesex University Hospital; Rebecca Lewis, Oladapo Fafemi, Jasdeep Gahir, Tasha Gandamihardja. Nottingham Breast Institute, Nottingham University Hospitals NHS Trust; Jennett Kelsall, Nazli Muhibullah, Charlene Otieno, Fayyaz Mazari, Marta Dauria, Lisa Whisker, Douglas Macmillan, Eleanor Gutteridge, Tuabin Rasheed, Hazem Khout, Kristjan Asgeirsson, Stephen McCulley. Ospedale Santa Chiara, University of Pisa, Italy; Maria Donatella Mariniello, Manuela Roncella, Matteo Ghilli, Livio Colizzi, Elena Rossetti, Lo Russo Marzia, Loredana Fustaino, Alessandro Quattrini Li. Oxford University Hospitals NHS Foundation Trust; Kate L Harvey, Rebecca Windle, Dionysios Dennis Remoundos, Pankaj Roy, Gael MacLean, Asha Adwani. Peterborough City Hospital, North West Anglia NHS Foundation Trust; Elena Popa, Steven Goh, Geeta Shetty. Poole Hospital NHS Foundation Trust; Sarah Clark. Portsmouth Hospitals NHS Trust; Lorenzo Bernaudo, Avi Agrawal, Lucy Mansfield. Princess Alexandra Hospital NHS Trust; Sally Tebbal, Ashraf Patel, Veronica Grassi. Queen Elizabeth Hospital Birmingham, University Hospitals Birmingham NHS Foundation Trust; Ojas Pujji, Kathryn Hamnett, Naren Basu. Royal Bolton Hospital, Bolton NHS Foundation Trust; Emily Granger, Michael Durbar, Panagiotis Pikoulas, Clare Garnsey, Philip Walker, Angela J Vollermere, loannis Michalakis. Royal Devon and Exeter NHS Foundation Trust; Robin Jones, Mina Youssef, Charlotte Ives, Mohammad Masood, Julie Dunn, Sisse Olsen, Douglas Ferguson, Rachel Tillett. Royal Free London NHS Foundation Trust; Anna Allan, Alex Woollard, Rebecca Canny, Alexander Woollard, Afshin Mosahebi, Stephen Hamilton, Shadi Ghali,Daniel Marsh, Jagdeep Chana, Nilesh Sojitra, Ibby Younis. Royal Hampshire County Hospital, Hampshire Hospitals NHS Foundation Trust; Dick Rainsbury, Natalie Chand, Vasileios Kalles, Anne Stebbing, Kevin Harris, Siobhan Laws. Royal Liverpool and Broadgreen University Hospitals NHS Trust; Chris Holcombe, Anne Tansley, Geraldine Mitchell, Emma de Sousa, Julia Henderson, Mysore Chandrashekar. Royal Marsden NHS Foundation Trust; Bernadette Pereira, Chloe Constantinou, Dalia Elfadl, Foivos 
Irakleidis, Izaro Hernan, Miriam Byrne, Natalie To, Rachel O'Connell, Jennifer Rusby, Peter Barry, Katerine Krupa, William Allum, Fiona MacNeill, Nicola Roche, Gerald Gui, Kelvin Ramsey, Paul Harris, Stuart James, Kieran Power. Royal United Hospitals Bath NHS Foundation Trust; Shelley Potter, Richard Sutton, Jamie Mclntosh, Nicola Laurence. Royal Victoria Infirmary, Newcastle Upon Tyne Hospitals NHS Foundation Trust; Louise MacLennan, Robert Milligan, Henry Cain, Adam Critchley, Joe O'Donoghue, Loraine Kalra, Nick Collis. Salisbury NHS Foundation Trust; Gina Weston-Petrides, Roanne Fiddes, Victoria Brown, Anna Aertssen, Diana SladeSharman, Mansoor Khan, Caroline McGuiness. Sant'Andrea Hospital of Rome; Vittoria Amorosi, Santanelli di Pompeo Fabio. St Bartholomew's Hospital, Barts Health NHS Trust; Georgios Exarchos, Natasha Jiwa, Jennifer Hu, Serena Ledwidge, Laura Johnson, Anthony Peel, Naseem Dhooma. St Vincent's University Hospital, Dublin; Eric Farrell, Liam Devane, Ruth Tevlin, Enda McDermott, Ruth Prichard, Denis Evoy, Jane Rothwell, James Geraghty, Colin Morrison, Catriona Lawlor. St. James University Hospital, The Leeds Teaching Hospitals NHS Trust; Fiona Langlands, Lauren Taylor, Philip Turton, Raj Achuthan, Kieran Horgan, Shireen Mckenzie, Brian Hogan, Mark Lansdown, Channegowda Navin. The Ipswich Hospital NHS Trust; Liz Sherwin, Caroline Mortimer, Neeraj Garg. The Mid Yorkshire Hospitals NHS Trust; Rahma Adam, Tahera Arif, Zbigniew Kryjak, Deedar Ali, Ravi Sowdi. The Royal Wolverhampton NHS Trust; Elena Fage, Senthurun Mylvaganam, Pilar Matey, Raghavan Vidya, Tapan Sircar. University Hospital of North Tees; Oubida Asaad, Pud Bhaskar, Matei Dordea. University Hospital of South Manchester; Ada Chrysafi, Damian McCartan, Rajiv Dave, Rachel Foster, Rebecca Wilson, Sylvia Okwemba, Yousef Majeed, Ciara O'Brien, Vinod Mathen, John Murphy, Nicola Barnes, Ashu Gandhi, James Harvey, Cliona C Kirwan, Richard Johnson. University Hospitals Coventry \& Warwickshire; Krupali Patel, Maria Dalmau Ribas, Natali Vigneswaran, Tom Challoner, Joanna Skillman, Alan Park, Maged Rizkalla, Abigail Tomlins, Kat McEvoy. University Hospitals of North Midlands NHS Trust; Sadaf Jafferbhoy, Soni Soumian, Sankaran Narayanan, Robert Kirby. West Hertfordshire Hospitals NHS Trust; Sladana Bajrusevic, Joseph Maalo, Michalis Charalambous, Lee Min Lai, Kelvin Chong, Simon Thomson, Sherif Monib. Whiston Hospital, St Helens and Knowsley Teaching Hospitals NHS Trust; Leena Chagla, Riccardo Audisio, Rieka Taghizadeh, Azhar Iqbal. Wirral University Teaching Hospital NHS Foundation Trust; Karen James, Maria Callaghan, Shabbir Poonawala, Jonathan Lund, Raman Vinayagam. Worcestershire Acute Hospitals NHS Trust; Sadaf Jafferbhoy, Steven Thrush, Rachel Bright Thomas, Michelle Mullan, Jevan Taylor. York Teaching Hospital NHS Foundation Trust; Ryo Yoshimura, Tom Mathew, Ben Mancey Jones, Kailas Munot, Rana Nasr, Jenny Piper, Deena El-Sharief. Zagazig University Hospital, Egypt; Mohammed Mustafa. Addenbrookes Hospital, Cambridge Universities NHS Foundation Trust; Dorin Dumitru. Airedale NHS Foundation Trust; Petros Christopoulos. Beaumont Hospital and the RCSI; Peter O'Leary. Chesterfield Royal Hospital NHS Foundation Trust; Irene Athanasiou. Edinburgh Breast Unit, Western General Hospital, NHS Lothian; Neil Johns. Homerton University Hospital NHS Foundation Trust; Disha Mehta. Milton Keynes university hospital NHS Foundation Trust; Saira Bibi, Farah Syed, Jacob Koris. Morriston Hospital, Abertawe Bro Morgannwg University Health Board; Samuel Healy, Lindsay Shanks. Musgrove Park Hospital, Taunton and Somerset NHS Foundation Trust; James Oliver. Nevill Hall Hospital, Aneurin Bevan University Health Board; Claudiu Bucata. North Middlesex University Hospital; Natalie Clarke. Nottingham Breast Institute, Nottingham University Hospitals NHS Trust; Kym Bignell, Malin Akerlund, Lisa Brock, Kelly Hallam. Portsmouth Hospitals NHS Trust; Rachel Howes, Alexander Armstrong, Claire Sethu. Royal Hampshire County Hospital, Hampshire Hospitals NHS Foundation Trust; Bashar Zeidan, Olivia Sjokvist. Royal Marsden NHS Foundation Trust; Marios Tasoulis. St Bartholomew's Hospital, Barts Health NHS Trust; Souganthy Sundaramoorthy. University Hospital of North Tees; Chad Harris, Dorothy Wintrip. Whiston Hospital, St Helens and Knowsley Teaching Hospitals NHS Trust; N Lymperopoulos, Yogesh Jain. Zagazig University Hospital, Egypt; Safa Ahmed Balata.

\section{AUTHOR CONTRIBUTIONS}

R.L.O., T.R. and R.V.D. contributed to the study design, data collection and interpretation of results; M.G. contributed to study design and coordinated data collection; A.T. performed the analysis, contributed to data interpretation and drafted the manuscript; J.S. and N.L.P.B. contributed to study design, data collection and interpretation, A.H. contributed to study design and interpretation; C.H. and S.P. conceived the study and led on the study design and conduct and the interpretation of the results. S.P. led on the analysis and wrote the first draft of the paper. All authors read and approved the final manuscript.

\section{ADDITIONAL INFORMATION}

Supplementary information is available for this paper at https://doi.org/10.1038/ s41416-019-0438-1.

Competing interests: The authors declare no competing interests.
Ethical approval and consent to participate: This study was classified as service evaluation by the UK National Health Service Research Authority Decision Tool (http://www.hra-decisiontools.org.uk/research/index.html), hence individual patient consent was not required. Local audit department approvals were obtained at each participating centre before commencing patient recruitment.

Data availability: The datasets generated during and/or analysed during the current study are not publicly available due to ongoing analyses but are available from the corresponding author on reasonable request.

Note: This work is published under the standard license to publish agreement. After 12 months the work will become freely available and the license terms will switch to a Creative Commons Attribution 4.0 International (CC BY 4.0).

Publisher's note: Springer Nature remains neutral with regard to jurisdictional claims in published maps and institutional affiliations.

\section{REFERENCES}

1. https://www.wcrf.org/int/cancer-facts-figures/data-specific-cancers/breastcancer-statistics. Accessed 16 April 2018 (2018).

2. Matala, C. M., McIntosh, S. A. \& Purushotham, A. D. Immediate breast reconstruction after mastectomy for cancer. Br. J. Surg. 87, 1455-1472 (2000).

3. Kummerow, K. L., Du, L., Penson, D. F., Shyr, Y. \& Hooks, M. A. Nationwide trends in mastectomy for early-stage breast cancer. JAMA Surg. 150, 9-16 (2015).

4. Harcourt, D. \& Rumsey, N. Psychological aspects of breast reconstruction: a review of the literature. J. Adv. Nurs. 35, 477-487 (2001).

5. Jeevan, R., Cromwell, D. A., Browne, J. P., Caddy, C. M., Pereira, J., Sheppard, C. et al. Findings of a national comparative audit of mastectomy and breast reconstruction surgery in England. J. Plast. Reconstr. Aesthet. Surg. 67, 1333-1344 (2014).

6. Harmeling, J., Kouwenberg, C., Bijlard, E., Burger, K., Jager, A. \& Mureau, M. A. M. The effect of immediate breast reconstruction on the timing of adjuvant chemotherapy: a systematic review. Breast Cancer Res. Treat. 153, 241 (2015).

7. Chavez-MacGregor, M., Clarke, C. A., Lichtensztajn, D. Y. \& Giordano, S. H. Delayed initiation of adjuvant chemotherapy among patients with breast cancer. JAMA Oncol. 2, 322-329 (2016).

8. Riba, L. A., Gruner, R. A., Fleishman, A. \& James, T. A. Surgical Risk Factors for the Delayed Initiation of Adjuvant Chemotherapy in Breast Cancer. Ann. Surg. Oncol. 25, 1904-1911 (2018).

9. Yu, K. D., Huang, S., Zhang, J. X., Liu, G. Y. \& Shao, Z. M. Association between delayed initiation of adjuvant CMF or anthracycline-based chemotherapy and survival in breast cancer: a systematic review and meta-analysis. BMC Cancer 13, 240 (2013).

10. Huang, J., Barbera, L., Brouwers, M., Browman, G. \& Mackillop, W. J. Does delay in starting treatment affect the outcomes of radiotherapy? A systematic review. J. Clin. Oncol. 21, 555-563 (2003).

11. Mikeljevic, J. S., Haward, R., Johnston, C., Crellin, A., Dodwell, D., Jones, A. et al. Trends in postoperative radiotherapy delay and the effect on survival in breast cancer patients treated with conservation surgery. Br. J. Cancer 90, 1343-1348 (2004).

12. Potter, S., Conroy, E. J., Williamson, P. R., Thrush, S., Whisker, L. J., Skillman, J. M. et al. The iBRA (implant breast reconstruction evaluation) study: protocol for a prospective multi-centre cohort study to inform the feasibility, design and conduct of a pragmatic randomised clinical trial comparing new techniques of implant-based breast reconstruction. Pilot Feasibility Stud. 2, 41 (2016).

13. Dave, R., O'Connell, R., Rattay, T., Tolkien, Z., Barnes, N., Skillman, J. et al. The iBRA2 (immediate breast reconstruction and adjuvant therapy audit) study: protocol for a prospective national multicentre cohort study to evaluate the impact of immediate breast reconstruction on the delivery of adjuvant therapy. BMJ Open 6, e012678 (2016)

14. Harris, P. A., Taylor, R., Thielke, R., Payne, J., Gonzalez, N., Conde, J. G. Research electronic data capture (REDCap)--a metadata-driven methodology and workflow process for providing translational research informatics support. J. Biomed. Inform. 42, 377-381 (2009).

15. Duxbury, P. J., Gandhi, A., Kirwan, C. C., Jain, Y. \& Harvey, J. R. Current attitudes to breast reconstruction surgery for women at risk of post-mastectomy radiotherapy: A survey of UK breast surgeons. Breast 24, 502-512 (2015).

16. Ho, A. Y., Hu, Z. I., Mehrara, B. J. \& Wilkins, E. G. Radiotherapy in the setting of breast reconstruction: types, techniques, and timing. Lancet Oncol. 18, e742-e753 (2017).

17. Jeevan, R., Mennie, J. C., Mohanna, P. N., O'Donoghue, J. M., Rainsbury, R. M., Cromwell, D. A. National trends and regional variation in immediate breast reconstruction rates. Br. J. Surg. 103, 1147-1156 (2016). 
The impact of immediate breast reconstruction on the time to delivery of... R.L. O'Connell et al.

18. Beecher, S. M., O'Leary, D. P., McLaughlin, R., Sweeney, K. J. \& Kerin, M. J. Influence of complications following immediate breast reconstruction on breast cancer recurrence rates. Br. J. Surg. 103, 391-398 (2016).

19. Mennie, J. C., Mohanna, P. N., O'Donoghue, J. M., Rainsbury, R. \& Cromwell, D. A. National trends in immediate and delayed post-mastectomy reconstruction procedures in England: a seven-year population-based cohort study. Eur. J. Surg. Oncol. 43, 52-61 (2017).

20. Ilonzo, N., Tsang, A., Tsantes, S., Estabrook, A. \& Thu, Ma. A. M. Breast reconstruction after mastectomy: a ten-year analysis of trends and immediate postoperative outcomes. Breast 32, 7-12 (2017).

21. Fischer, J. P., Nelson, J. A., Au, A., Tuggle, C. T. 3rd, Serletti, J. M. \& Wu, L. C. Complications and morbidity following breast reconstruction-a review of 16,063 cases from the 2005-2010 NSQIP datasets. J. Plast. Surg. Hand Surg. 48, 104-114 (2014).

22. Mylvaganam, S., Conroy, E., Williamson, P. R., Barnes, N. L. P., Cutress, R. I. \& Gardiner, M. D. et al. Variation in the provision and practice of implant-based breast reconstruction in the UK: Results from the iBRA national practice questionnaire. Breast 35, 182-190 (2017).

23. Dikmans, R. E., Negenborn, V. L., Bouman, M. B., Winters, H. A., Twisk, J. W., Ruhe, P. Q. et al. Two-stage implant-based breast reconstruction compared with immediate one-stage implant-based breast reconstruction augmented with an acellular dermal matrix: an open-label, phase 4, multicentre, randomised, controlled trial. Lancet Oncol. 18, 251-258 (2017).

24. Wilkins, E. G., Hamill, J. B., Kim, H. M., Kim, J. Y., Greco, R. J., Qi, J. et al. Complications in postmastectomy breast reconstruction: one-year outcomes of the mastectomy reconstruction outcomes consortium (MROC) study. Ann. Surg. 267, 164-170 (2018)
25. Potter, S., Conroy, E. J., Cutress, R. I., Williamson, P. R., Whisker, L., Thrush, S. et al Short-term safety outcomes of mastectomy and immediate implant-based breast reconstruction with and without mesh (iBRA): a multicentre, prospective cohort study. Lancet Oncol. 20, 254-266 (2019).

26. Hasak, J. M., Myckatyn, T. M., Grabinski, V. F., Philpott, S. E., Parikh, R. P., Politi, M. C. Stakeholders' perspectives on postmastectomy breast reconstruction: recognizing ways to improve shared decision making. Plast. Reconstr. Surg. Glob. Open $\mathbf{5}$, e1569 (2017).

27. Browne, J. P., Jeevan, R., Gulliver-Clarke, C., Pereira, J., Caddy, C. M. \& van der Meulen, J. H. P. The association between complications and quality of life after mastectomy and breast reconstruction for breast cancer. Cancer 123, 3460-3467 (2017).

28. Fischer, J. P., Fox, J. P., Nelson, J. A., Kovach, S. J. \& Serletti, J. M. A longitudinal assessment of outcomes and healthcare resource utilization after immediate breast reconstruction-comparing implant- and autologous-based breast reconstruction. Ann. Surg. 262, 692-699 (2015).

29. di Summa, P. G., Tay, S. K., Stevens, R., Doughty, J. C. \& Bramhall, R. J. Neoadjuvant radiotherapy (NART) in breast reconstruction - The future for autologous reconstruction in locally advanced disease? J. Plast. Reconstr. Aesthet. Surg. 71, 935-937 (2018).

30. Curigliano, G., Criscitiello, C., Esposito, A. \& Pruneri, G. Over-using chemotherapy in the adjuvant setting. Breast 31, 303-308 (2017).

31. Russell, N. S., Kunkler, I. H. \& van Tienhoven, G. Determining the indications for post mastectomy radiotherapy: moving from 20th century clinical staging to 21 st century biological criteria. Ann. Oncol. 26, 1043-1044 (2015). 